\title{
Theoretical studies on the transmission and reflection properties of metallic planar fractals
}

\author{
Lei Zhou, C T Chan and Ping Sheng \\ Department of Physics, The Hong Kong University of Science and Technology, \\ Clear Water Bay, Kowloon, Hong Kong, People's Republic of China \\ Received 27 May 2003 \\ Published 14 January 2004 \\ Online at stacks.iop.org/JPhysD/37/368 (DOI: 10.1088/0022-3727/37/3/009)
}

\begin{abstract}
We describe a systematic finite-difference-time-domain study on the electromagnetic (EM) characteristics of a specific type of planar metallic fractal patterns. These fractals are shown to totally reflect EM waves at a series of frequencies; between any two reflection frequencies there can be total transmission at some particular frequency. We show that both reflection and transmission are induced by local resonances, and develop an effective medium model to reproduce the simulation results. A double stack of $90^{\circ}$-rotated fractals is shown to exhibit absolute band gaps independent of both the polarization and incidence angles. In particular, the size of the band gaps can be significantly enlarged by multilayer stacking, and the fractal sample size can be significantly smaller than the relevant band gap wavelength.
\end{abstract}

\section{Introduction}

Photonic band gap (PBG) materials can reflect electromagnetic (EM) waves at selected frequency regimes for arbitrary incidence angles. Conventional PBG materials are conceived and constructed as a periodic array of dielectrics, employing the mechanism of Bragg scattering to generate a forbidden band gap [1]. All real photonic crystals are finite in size. In order to function effectively, the thickness and lateral dimensions of a photonic crystal slab must be a few times that of the wavelength. This restriction, a natural consequence of the Bragg mechanism, makes photonic crystals too bulky for long wavelength applications, i.e. in the microwave and radiowave regimes. For example, the wavelength corresponding to a $1 \mathrm{GHz}$ EM wave is $30 \mathrm{~cm}$, and a few times the wavelength would be over $1 \mathrm{~m}$. Frequency selective surfaces (FSS) [2], consisting of a periodic array of metallic elements arranged on a surface, can also selectively reflect EM waves at some pre-specified frequency. It operates on the resonance principle and therefore can be thin, but the corresponding wavelength for reflection is still bounded by the lateral size of the unit element. A large surface is required to reflect low frequency EM waves.

These restrictions can be lifted by a particular class of planar fractal patterns [3]. Fractals have been exploited in multi-band and reduced-sized antennas, and their diffraction properties have been studied ([4] and references therein). Recently, we reported that a class of space-filling curves could be used to construct planar frequency selective materials with multiple band gaps for EM waves over an ultra-wide frequency regime [5]. In particular, these metallic fractals were able to reflect EM waves at wavelength much larger than the sample size [6]. In this paper, we give a detailed theoretical study on the metallic fractals $[5,6]$ through finitedifference-time-domain (FDTD) simulations [7,8]. Below, we describe our theoretical approach in section 2, discuss the underlying physics of the reported phenomena in section 3, and demonstrate in section 4 through FDTD simulation that a double stack of fractals can show absolute band gaps and the gap width can be significantly enlarged by multilayer stacking, and summarize our results in section 5 .

\section{Computational methodology}

For our FDTD simulations ${ }^{1}$, we first construct an artificial rectangular waveguide placed along the $z$-axis. Perfect electric conducting (PEC) boundary conditions $(\vec{n} \times \vec{E}=0, \vec{n} \cdot \vec{B}=0)$

1 Simulations were performed using a commercial software package CONCERTO 2.0, developed by Vector Fields Limited, England, 2001. 
are imposed on the two walls parallel to the $x z$ plane, and perfect magnetic conducting (PMC) boundary conditions $(\vec{n} \cdot \vec{E}=0$, $\vec{n} \times \vec{B}=0$ ) imposed on the other two (see figure $1(a)$ ). This artificial waveguide corresponds exactly to a square subset of an infinite parallel-plate transmission line. By placing the metallic fractal inside the waveguide, one can perform the standard S-parameter calculations. It is easy to verify that the TEM mode of such an artificial waveguide corresponds to a plane wave. By specifying the wave templates of both the input and the output ports to be a TEM mode, we calculate the zeroorder transmission/reflection properties of the object. The walls of our artificial waveguide introduce an infinite number of object images so that our model system, when translated to a realistic situation, corresponds to an infinite plane tiled by a periodic replica of the object under study.

Convergence of our calculations was tested against the discretization adopted in FDTD simulations [7, 8]. Figure 1(b) compares the reflection spectra of a 4-level metallic fractal pattern calculated using different meshes in the FDTD simulations. In our simulations, maximum cell size is adopted in homogeneous regimes (say, air, or inside the bulk dielectrics). Finer sub-meshes are adopted in space regions where strong inhomogeneity exists (say, near the air/metal interface). In particular, a metallic object is divided into at least two parts (along each direction) in order to ensure

(a)

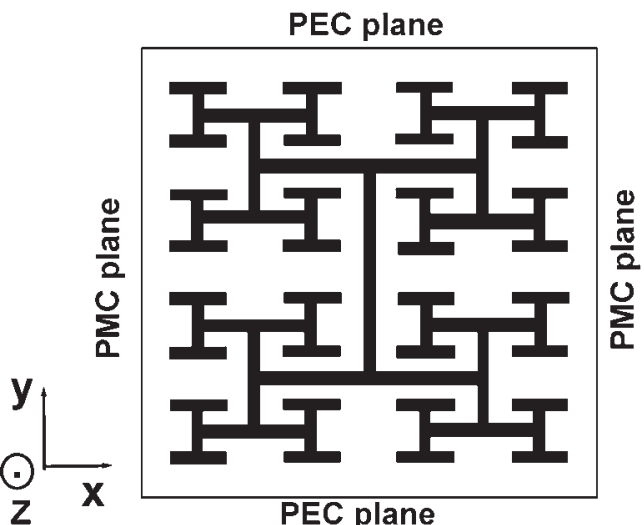

(b)

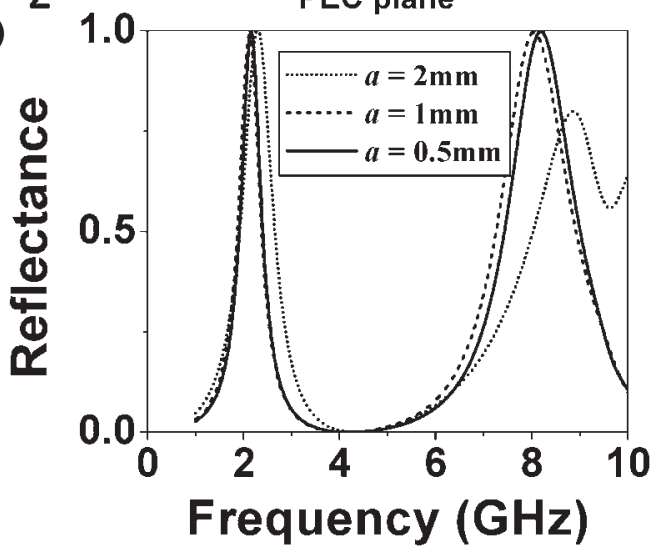

Figure 1. (a) Schematic illustration of the geometry that we study - a planar metallic fractal of thin but finite thickness (along the $z$ direction) is put inside an artificial waveguide described in the text. (b) Reflectance as a function of frequency for a 4-level metallic fractal, calculated by FDTD simulations using cubic meshes with maximum unit size of $a^{3}$. Fractal details: first level line length $=16 \mathrm{~mm}$, line width and thickness $=0.2 \mathrm{~mm}$. the converged results. It is clear that using a cubic mesh with maximum cell size $1 \mathrm{~mm} \times 1 \mathrm{~mm} \times 1 \mathrm{~mm}$ is good enough to get convergent results for frequencies $<10 \mathrm{GHz}$. Higher frequencies require finer meshes. In addition, in a dielectric media the mesh should be denser than that in vacuum because the wavelength is scaled by the refractive index. Convergence should be carefully tested in each case. However, our experience has been that dividing the shortest metal lines to eight parts was enough to get convergent results in the relevant frequency regimes.

\section{Results and the underlying physics}

Limited by our computational power, it is difficult to directly study the 15-level fractal plate adopted in the experiment [5], since the number of mesh points required is prohibitively large for an accurate simulation. Fortunately, calculations reveal that certain frequencies would excite only particular subdomains of the fractal. With increasing frequency, the relevant characteristics are governed by localized currents in higher level sub-structures. Figure 2 compares the FDTD results for a periodically replicated 6-level fractal and a periodically replicated 4-level fractal, with the latter being exactly a substructure of the 6-level fractal. Both simulations adopted the same mesh size to discretize the model. While the 4-level calculation fails to reproduce the low-frequency features of its mother structure, which was determined by the response of the whole 6-level structure, it yields almost the identical results with the 6-level calculation at the high frequency regime where the physics was governed by currents localized in the sub-structures.

This fact enables us to use the technique of 'divide and conquer' to calculate the response of a large system. We first apply a coarse discretization to calculate the response of the whole fractal (periodically replicated), yielding the low frequency characteristics involving long current paths in the fractal structure. But the results are inaccurate for the high frequencies. To determine the accurate higher frequency characteristics, we focus only on the higher level sub-structures (also periodically replicated), but with finer discretization. With this approach, converged results for the experimentally adopted 15-level fractal plate [5] were obtained. These are shown as open symbols in figures 3 and 4 .

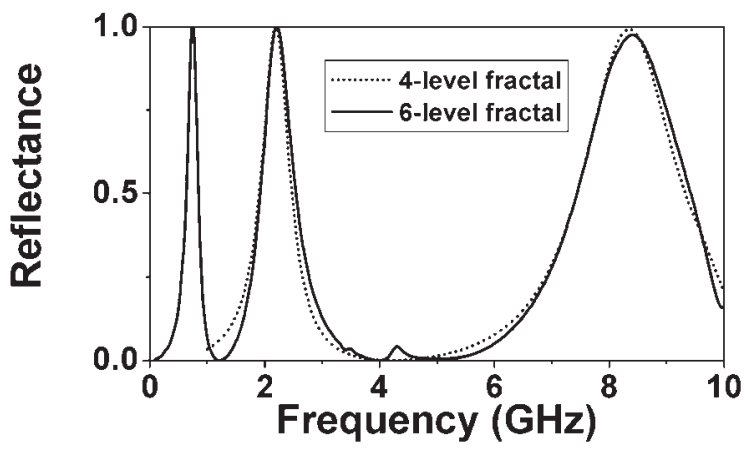

Figure 2. FDTD simulated reflectance as a function of frequency for a 6-level fractal (first level: $32 \mathrm{~mm}$ ) and a 4 level fractal (first level $16 \mathrm{~mm}$ ) under the same discretization mesh with $a=1 \mathrm{~mm}$. 


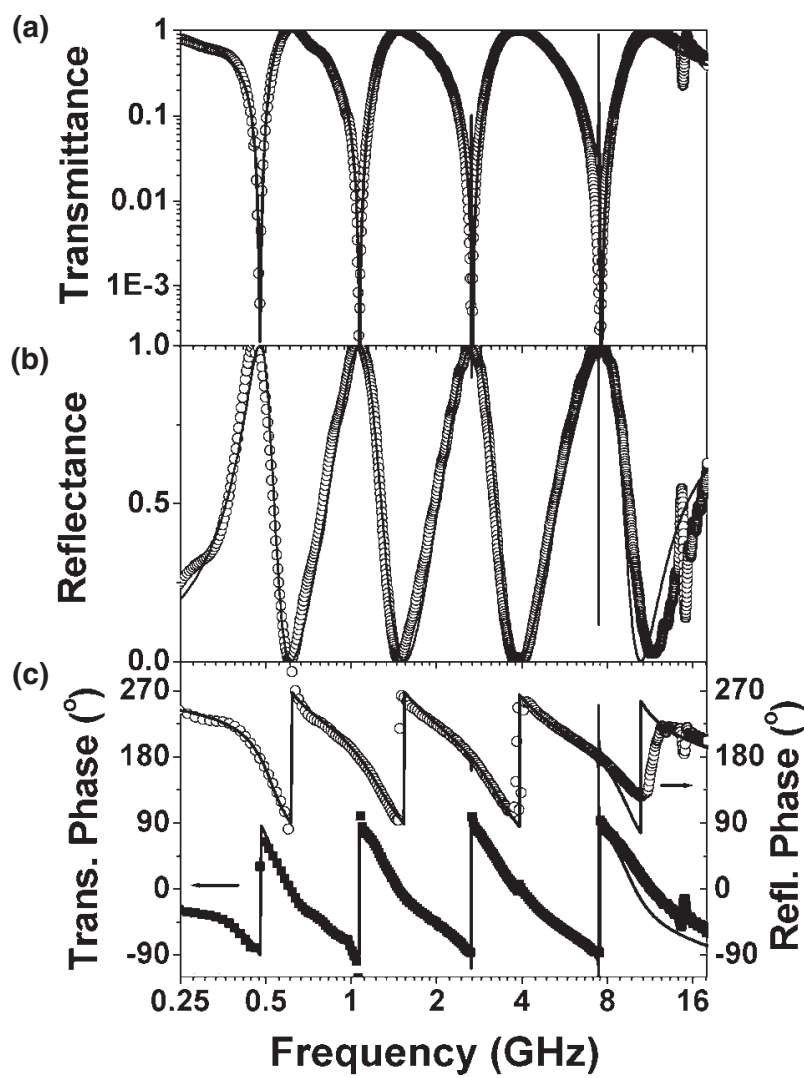

Figure 3. Transmittance $(a)$, reflectance $(b)$, transmission phase change (across the sample) and reflection phase change (measured on the top surface) (c) for a 15-level fractal plate (first level: $144.78 \mathrm{~mm}$, line width and thickness $=0.1 \mathrm{~mm}$, $1.0 \mathrm{~mm}$-thick dielectric substrate with $\varepsilon=5.3$ ) with respect to an $x$-polarized normally incident wave ( $\vec{E}$ perpendicular to the first level line). Symbols denote FDTD simulation results; solid lines the effective medium model as described in the text.

The low $(0.1-0.9 \mathrm{GHz})$, mid $(0.9-2.2 \mathrm{GHz})$ and high frequency $(2.2-18 \mathrm{GHz})$ results were calculated (for the $y$-polarization) from periodic replica of 11-, 9- and 7-level substructures of the 15 -level fractal, respectively. For the $x$-polarization, we also used 11-, 9-, 7-level sub-structures to calculate the low $(0.1-0.6) \mathrm{GHz}$, mid (0.6-1.4) and high frequency (1.4-18) spectra, respectively. The basic cell (with maximum cell size) is taken to be $(a / 2, a / 4, a / 4)$ for the 11-level system, $(a / 4, a / 4, a / 8)$ for the 9-level one, and $(a / 8, a / 8, a / 8)$ for the 7-level one, where $a=1.1311 \mathrm{~mm}$ is the length of the highest level lines. There is exactly the same number of resonances as the number of levels in a fractal. Hence, there are more resonances at lower and higher frequencies than those shown in figures 3 and 4, but those are beyond our computational capabilities for an accurate answer. We see that the calculated spectra have quasi-log-periodicity. This is a manifestation of the fractal's geometric self-similarity. The log-periodicity of the spectra is not exact for the present fractal structure with a finite number of levels, but the spectra will approach log-period behaviour as the number of levels increases. Log-periodicity also implies that the resonance frequencies (hence the stop bands) cover an ultrabroad range, in contrast to the conventional PBG slabs and (a)

(b)

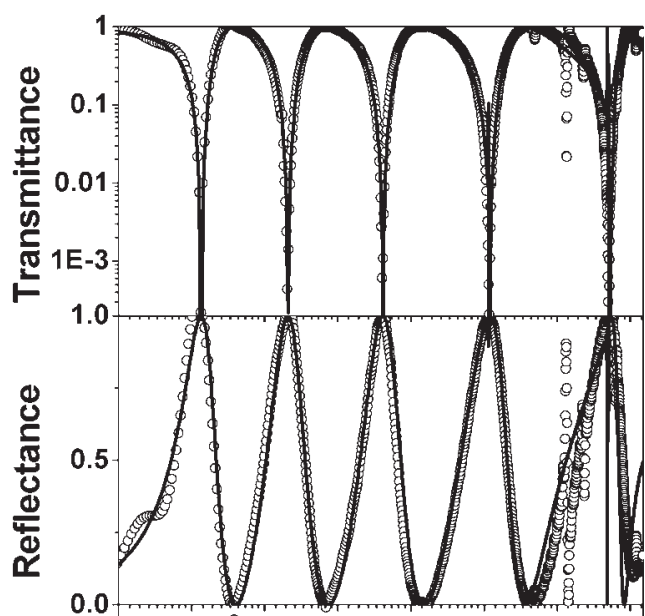

(c)

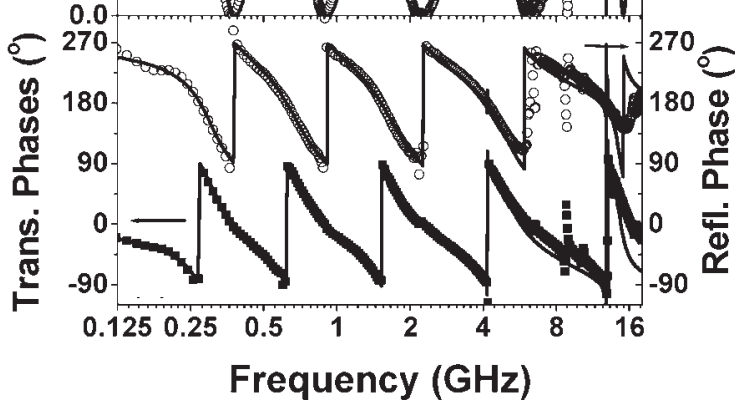

Figure 4. Simulation results (denoted by symbols), and effective medium predictions (denoted by solid lines) for the $y$-polarization case. Parameter values are the same as those for figure 3 .

FSS which typically have one single dominant response, and high harmonics typically cover a linear scale in the frequency spectrum.

An analysis of the simulation results offers us a picture of the underlying physics. The EM field is found to excite currents in the sub-structure of the metallic lines of the fractal, with the current amplitude reaching a maximum at those frequencies where the fractal is strongly reflecting. The relative phase of the current with respect to the input wave undergoes a $\pi$ jump whenever the frequency is varied across each maximum reflection point, indicating a resonance behaviour. Each resonance has currents excited mainly along the metal lines of one specific level, flowing towards the higher (finer) level structures. As an illustration, the amplitude of the surface current density distribution (calculated by $\vec{J}=n \times \vec{H}$ ) is shown in figure $5(a)$ for the resonance at $4.2 \mathrm{GHz}$, and in figure $5(b)$ for the resonance at $13.1 \mathrm{GHz}$. The current amplitude is seen to peak at the 11th level lines for resonance frequency $4.2 \mathrm{GHz}$ and to peak at the 13th level lines for the $13.1 \mathrm{GHz}$ resonance, each flowing to higher level lines on both sides. It is known that the dielectric constant of a dispersive medium with localized resonances takes a general form $\varepsilon(f)=a+\sum_{j=1}^{N} b_{j} /\left(f_{j}^{2}-f^{2}\right)$, where $f_{j}$ is the $j$ th resonance frequency, $f$ is the frequency, and $a, b_{j}$ are some model parameters [9]. Adjusting parameters $a, b_{j}$ to fit our calculation results, we find that the fractal response at normal incidence can be accurately modelled by a thin homogeneous plate (of the same thickness of the real plate $1.1 \mathrm{~mm}$ ) with an 
(a)

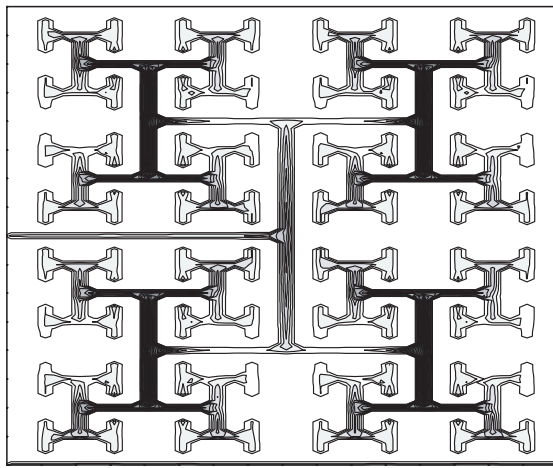

(b)

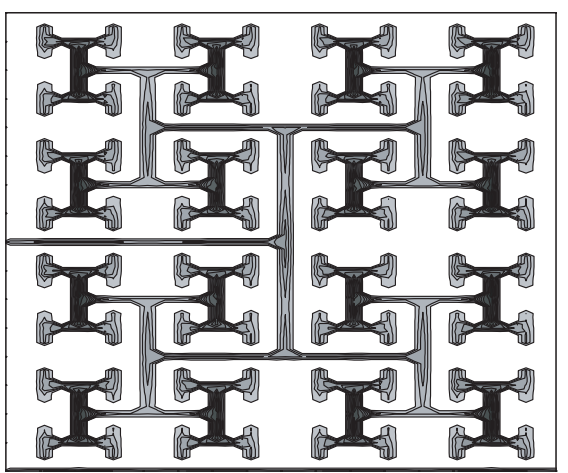

Figure 5. Surface current density (in $\mathrm{A} \mathrm{mm}^{-1}$ ) distribution on the fractal surface under an incident EM wave with frequency $4.2 \mathrm{GHz}(a)$ and $13.1 \mathrm{GHz}(b)$.

effective dielectric constant of the form:

$$
\begin{aligned}
\varepsilon_{x x}(f) & =11+\frac{15}{0.477^{2}-f^{2}}+\frac{48}{1.07^{2}-f^{2}} \\
+ & \frac{130}{2.66^{2}-f^{2}}+\frac{470}{7.55^{2}-f^{2}}, \\
\varepsilon_{y y}(f) & =11+\frac{10}{0.274^{2}-f^{2}}+\frac{23}{0.623^{2}-f^{2}} \\
+ & \frac{60}{1.53^{2}-f^{2}}+\frac{190}{4.19^{2}-f^{2}}+\frac{500}{13.06^{2}-f^{2}},
\end{aligned}
$$

where $f$ denotes the frequency measured in gigahertz. Solid lines in figures 3 and 4 are the results calculated based on such an effective medium. Excellent agreement with the FDTD results is noted for not only the amplitudes but also the phases of the transmission/reflection coefficients. The effective medium model also offers us an explanation for the total transmissions, which appeared at first sight to be particularly intriguing since the metallic lines in the fractal pattern are finely separated (much smaller than the relevant wavelength). In between any two total reflection frequencies, there is a frequency at which the currents induced on two adjacent resonance structures are roughly of the same magnitude but $180^{\circ}$ out of phase, leading to a cancellation of their radiations in far-field. A metallic structure with induced currents effectively cancelling each other (seen in far field) would yield no metallic blocking effect to the incident EM wave, since it is similar to an insulating dielectric. It is worth noting that the reflected wave has a $180^{\circ}$ phase change with respect to the incident wave at each total reflection point, similar to the case of a perfect metal.

\section{Absolute band gaps}

From figures 3 and 4 it is seen that the total reflection frequencies are dependent on the polarization of the incidence EM wave. Hence, they cannot simulate the effect of an absolute stop gap. However, by using a 3-level fractal structure as an example we demonstrate that a perpendicular stack of two identical fractal plates, with the second one rotated by $90^{\circ}$ (with respect to the plate's normal direction) relative to the first, can exhibit total reflections independent of both the incidence angle and the polarization. Therefore, such double stacks simulate reflection from absolute band gaps. It is somewhat tricky to study the transmission/reflection properties under off-normal incidence angles. Below, we describe an approach using the FDTD simulations. In the waveguide that we constructed, higher order modes (rather than the TEM mode that we used to simulate the plane wave) exist, whose wave forms can be easily calculated. For example, by following the standard procedure adopted for a conventional waveguide [9], a $\mathrm{TE}_{m n}$ mode has the following field templates:

$$
\begin{gathered}
E_{x}=-\mathrm{i} k_{y}\left(\frac{c k}{k_{c}^{2}}\right) B_{0} \sin \left(k_{x} x\right) \sin \left(k_{y} y\right) \mathrm{e}^{\mathrm{i}\left(k_{z} z-\omega t\right)}, \\
E_{y}=-\mathrm{i} k_{x}\left(\frac{c k}{k_{c}^{2}}\right) B_{0} \cos \left(k_{x} x\right) \cos \left(k_{y} y\right) \mathrm{e}^{\mathrm{i}\left(k_{z} z-\omega t\right)}, \\
E_{z}=0, \\
B_{x}=\mathrm{i} k_{x}\left(\frac{k_{z}}{k_{c}^{2}}\right) B_{0} \cos \left(k_{x} x\right) \cos \left(k_{y} y\right) \mathrm{e}^{\mathrm{i}\left(k_{z} z-\omega t\right)}, \\
B_{y}=\mathrm{i} k_{y}\left(\frac{k_{z}}{k_{c}^{2}}\right) B_{0} \sin \left(k_{x} x\right) \sin \left(k_{y} y\right) \mathrm{e}^{\mathrm{i}\left(k_{z} z-\omega t\right)}, \\
B_{z}=B_{0} \sin \left(k_{x} x\right) \cos \left(k_{y} y\right) \mathrm{e}^{\mathrm{i}\left(k_{z} z-\omega t\right),}
\end{gathered}
$$

where $k_{x}=m \pi / L_{x}, k_{y}=n \pi / L_{y}, k_{c}=\sqrt{k_{x}^{2}+k_{y}^{2}}, k_{z}=$ $\sqrt{(\omega / c)^{2}-k_{x}^{2}-k_{y}^{2}}$, and $L_{x}$ and $L_{y}$ are the lateral dimensions of the waveguide. It is rather straightforward to demonstrate that equation (2) is a linear combination of four linearly polarized plane waves travelling along four directions: $\vec{F}(\vec{r})=$ $\sum_{j=1}^{4} \vec{F}_{j} \mathrm{e}^{\mathrm{i}\left(\vec{k}_{j} \cdot \vec{r}\right)}$ in which $\vec{k}_{j}= \pm k_{x} \hat{x} \pm k_{y} \hat{y}+k_{z} \hat{z}$ and $\vec{F}(\vec{r})=$ $[\vec{E}(\vec{r}), \vec{H}(\vec{r})]$. The total transmitted wave should be generally $\vec{F}^{\mathrm{t}}(\vec{r})=\sum_{j=1}^{4} t_{j} \vec{F}_{j} \mathrm{e}^{\mathrm{i}\left(\vec{k}_{j} \cdot \vec{r}\right)}$, where $t_{j}$ is the transmission coefficient for the $j$ th input plane wave. If the system under study has reflection invariance symmetry (with respect to both the $x z$ and the $y z$ planes), the transmission coefficient for the four $\vec{k}$ vectors $\left(\vec{k}_{j}= \pm k_{x} \hat{x} \pm k_{y} \hat{y}+k_{z} \hat{z}\right)$ must be the same (i.e. $t_{j}=t$ ), so that the total transmitted wave becomes $\vec{F}^{\mathrm{t}}(\vec{r})=t \sum_{j=1}^{4} \vec{F}_{j} \mathrm{e}^{\mathrm{i}\left(\vec{k}_{j} \cdot \vec{r}\right)}=t \vec{F}(\vec{r})$. It is clear that transmission/reflection coefficients calculated with respect to a waveguide mode specified in equation (2) are the same as those calculated with respect to a plane wave with one of the $\vec{k}_{j}$ 's. Since $\vec{k}_{j}$ has non-zero transverse component, we thus formulated an approach to study the oblique incident angle case using FDTD simulation. Varying $L_{x}$ and $L_{y}$ (by varying the number of unit structures contained in the object put inside the waveguide), we can tune the values of $k_{x}$ and $k_{y}$ so as to effectively modify the incidence angle. The incidence angle can also be changed by selecting different modes specified 
(a)

(b)
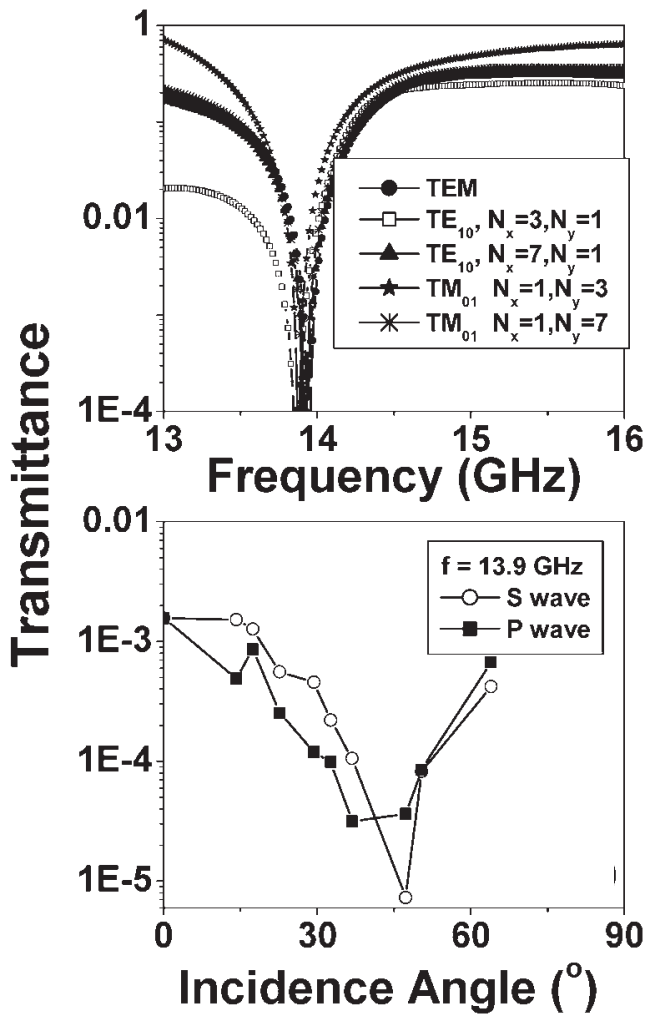

Figure 6. (a) Transmission spectra of a double stack of 3-level fractal under different input waveguide modes. $N_{x}$ and $N_{y}$ indicate the number of unit cells included in the object under investigation. Unit cell details: first level length $=2 \mathrm{~mm}$, line width and thickness $=0.1 \mathrm{~mm}$, inner dielectric layer thickness $=1.0 \mathrm{~mm}$ $(\varepsilon=5.3)$, and total lateral size of the unit measures $4 \mathrm{~mm} \times 4 \mathrm{~mm}$; the first 3-level fractal is attached to the front plane of the dielectric layer, while the second 3-level fractal plate, rotated by $90^{\circ}$ relative to the first, is attached to the back plane of the dielectric layer.

(b) Transmittance as a function of the incidence angle at $13.9 \mathrm{GHz}$ for the S-polarized incident wave $(\vec{E} \| \hat{y})$ and the P-polarized one $(\vec{H} \| \hat{x})$.

by the numbers $n$ and $m$. The same arguments apply also to the $\mathrm{TM}_{n m}$ modes, which differ from the corresponding $\mathrm{TE}_{n m}$ modes only in their polarizations.

We compare the transmission spectra for a double stack of 3-level fractals, for different input waveguide modes corresponding to different fixed transverse $\vec{k}$ components. We emphasize here that a spectrum remains exactly the same if we rotate the double-stack by $90^{\circ}$ so that the rotated spectrum is not plotted. The most important feature to be noted is that the resonance frequencies, and thus the gap positions, coincide well. This is a direct demonstration of the absolute band gap effect, since different transverse $k$ components correspond to different incidence angles at a fixed frequency. Selecting a frequency $(13.9 \mathrm{GHz})$ inside the gap, we plotted the transmission as a function of incidence angle in figure $6(b)$ for both the S-polarized and the P-polarized incident waves. The transmittance is seen to be well below $20 \mathrm{~dB}$ for all the oblique incidence angles that we have calculated.

The size of the stop bands can be significantly enhanced by stacking identical fractals together. Figure 7 compares the calculated transmission spectra of a single fractal, two identical fractals, and four identical fractals stacked together. The

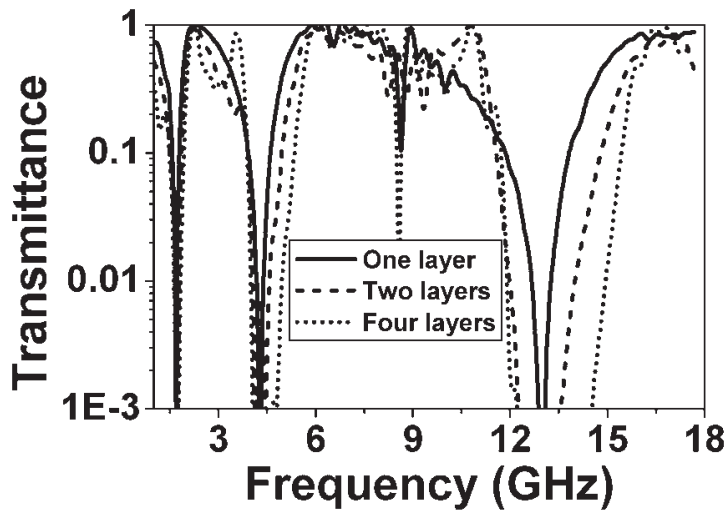

Figure 7. FDTD calculated transmission spectra for a single fractal, two identical fractals, and four identical ones stacked together, each separated by a $1 \mathrm{~mm}$ air gap from its neighbours. The fractal plate is the same as that described in figures 3 and 4.

fractals are not rotated, and they are separated from each other by a $1 \mathrm{~mm}$ air gap. The stop bands are visibly expanded (there is also a slight shift in midgap frequencies). Since the thickness is not an issue as long as each fractal is thicker than the skin depth, a stack of several fractals, plus the corresponding $90^{\circ}$ rotated counterparts, can still be regarded as thin compared to the relevant wavelengths. The stop bands can also be expanded by increasing the width of metal lines in the fractal.

\section{Conclusions}

To summarize, we have presented a systematic study on the EM wave characteristics of a specific type of metallic planar fractal through FDTD simulations. We find such metallic fractals to exhibit multiple total reflections and total transmission characteristics, whose physics is governed by a series of self-similar local resonances. The fractal pattern can be well described as an effective-medium model with a dispersive dielectric constant. We demonstrated through the FDTD simulations that a double stack of fractals, with the second one rotated by $90^{\circ}$ with respect to the first one, possesses absolute PBGs with total reflection independent of both the polarization and incidence angles. We also showed that the sizes of the band gaps could be significantly enlarged by multilayer stacking. In addition, all the effects described above can be achieved with samples significantly smaller than the relevant wavelength.

\section{Acknowledgments}

This work is supported by RGC Hong Kong through HKUST6138/00P

\section{References}

[1] Yablonovitch E 1987 Phys. Rev. Lett. 582059 John S 1987 Phys. Rev. Lett. 582486 Joannopoulos J D, Meade R D and Winn J 1995 Photonic Crystals (Princeton, NJ: Princeton University Press)

[2] Munk B A 2000 Frequency Selective Surfaces, Theory and Design (New York: Wiley)

[3] Barnsley M 1988 Fractal Everywhere (San Diego: Academic) 
EM characteristics of planar metallic fractals

[4] Werner D H and Mittra R 2000 Frontiers in Electro (New York: IEEE Press)

[5] Wen W, Zhou L, Li J, Ge W, Chan C and Sheng P 2002 Phys. Rev. Lett. 89223901

[6] Zhou L, Wen W, Chan C and Sheng P 2003 Appl. Phys. Lett. 821012
[7] Yee K S 1966 IEEE Trans. Antennas and Propagation 14302

[8] Taflove A and Hagness S C 2000 Computational Electrodynamics: The Finite-Difference Time-Domain Method (Norwood: Artech House)

[9] Jackson J D 1975 Classical Electrodynamics (New York: Wiley) 